\title{
Loss aversion, large deviation preferences and optimal portfolio weights for some classes of return processes
}

\author{
Ken Duffy ${ }^{(1, *)}$, Olena Lobunets ${ }^{(2)}$ and Yuri Suhov ${ }^{(3)}$
}

August 2006; Revised November 2006

(1) Hamilton Institute, National University of Ireland, Maynooth, Ireland
(*) Phone:+353-1-7086463. Fax:+353-1-7086269. E-mail: ken.duffy@nuim.ie
(2) University of Essex, Colchester, UK
(3) Statistical Laboratory, DPMMS, Cambridge University, Cambridge, UK

\begin{abstract}
We propose a model of a loss averse investor who aims to maximize his expected wealth under certain constraints. The constraints are that he avoids, with high probability, incurring a (suitably defined) unacceptable loss. The methodology employed comes from the theory of large deviations. We explore a number of fundamental properties of the model and illustrate its desirable features. We demonstrate its utility by analyzing assets that follow some commonly used financial return processes: Fractional Brownian Motion, Jump Diffusion, Variance Gamma and Truncated Lévy.
\end{abstract}

PACs: 89.65.Gh; 02.50.-r; 05.40.-a. MSC: 91B28; 62P20.

Keywords: portfolio selection; loss averse investors; large deviations approach.

\section{Introduction}

Risk averse investors are often characterized by a utility function that encodes the values the investor places on returns from a financial object. With risk measured in terms of a radical of the return process, risk aversion is then measured as the marginal reward an investor needs to receive if he takes a given amount of risk.

In this paper we treat a different paradigm. We consider a loss averse investor whose aim is to invest in a static, single-period portfolio that maximizes his expected return at a trading time scale while safeguarding, with high probability, the return from falling below an acceptable level. In this setting an investor is defined by the trading time scale, the threshold that 
determines the unacceptable loss and a specified bound on the probability that such a loss occurs.

Questions regarding a static portfolio selection, by investors who update their portfolio's composition infrequently, are of considerable practical interest. For example, the articles [1], [2], and references therein, present strong evidence that when US employees invest in pensions they typically do so with the employer's recommended portfolio composition and do not review their options afterwards. Moreover, the article [3] reports that this composition is often not changed over time by the employer either.

Here we consider a number of constraints: (1) the investor only cares if his portfolio's value is less than the unacceptable threshold at the trading time scale; (2) the investor wishes the portfolio value not to fall below the unacceptable loss at certain observation times prior to the trading time; (3) the investor wishes the portfolio value not to fall below the unacceptable loss at any stage in the future.

The main object of our analysis is the so-called large deviation preferences function (LDPF) introduced in section 2. This function succinctly encapsulates the stochastic nature of the return process and enters naturally into probabilistic bounds. The LDPF is mathematically analogous to the effective bandwidth function introduced during the early '90s in the study of telecommunications systems (see, for example, Kelly [4]) and closely related to the free energy in statistical mechanics, as will be explained in the next section. In section 3 we introduce bounds that enable the investor to identify portfolios satisfying a given constraint, from which he may choose the one that has the maximum expected return. In section 4 we calculate explicitly the LDPF for certain commonly used return processes. In sections 5 and 6 we present some examples of portfolios based on the model.

\section{Large deviation preferences}

Let $X(t)$ denote the monetary value of a financial object at time $t \geq 0$ and assume that the random process $\{X(t), t \geq 0\}$ has stationary increments, with all moments $\mathbb{E}|X(t)|^{n}<\infty$. We define the LDPF $U_{\mathrm{LD}}$ (for the process $\{X(t)\}$ ) as a function

$$
(s, t) \in \mathbb{R} \times[0,+\infty) \mapsto U_{\mathrm{LD}}(s, t) \in \mathbb{R} \cup\{-\infty,+\infty\}
$$

given by

$$
U_{\mathrm{LD}}(s, t)=-\frac{1}{s t} \log E(\exp (-s X(t))) \text { for } s \neq 0 \text { and } U_{\mathrm{LD}}(0, t)=\lim _{s \downarrow 0} U_{\mathrm{LD}}(s, t) .
$$

Here $t$ is a time scale and $s$ is a value scale $\left(s^{-1}\right.$ is expressed in monetary units, and $U_{\mathrm{LD}}(s, t)$ has units of money per time $\left.{ }^{1}\right)$. If, for a given $t$, the function $s \mapsto U_{\mathrm{LD}}(s, t)$ is finite for

\footnotetext{
${ }^{1}$ For a Gaussian return process, the parameter $s$ gives the coefficient of relative risk aversion; cf. Equation (5) below.
} 
$-s^{-}<s<s^{+}$where $s^{-}<0, s^{+}>0$, then it completely specifies the distribution of the random variable $X(t)$ (see, for example, Billingsley [5]). Furthermore, $U_{\mathrm{LD}}(s, t)$ is then infinitely differentiable with respect to $s$ in the interval $\left(-s^{-}, s^{+}\right)$.

The LDPF of a financial object is motivated by the free energy and entropy in Statistical Mechanics. With $X$ denoting the energy level of a system, its free energy is defined to be $-\beta^{-1} \log \mathbb{E}(\exp (\beta X))$, where $\beta$ is inverse temperature and the expectation is taken over a probabilistic ensemble of energy levels. For fixed time, this suggests identifying $\beta$ with $s$, so that monetary value plays the role of temperature. For financial objects it is natural to look at collection of systems indexed by time. As we expect value to have a natural drift with time, we re-scale the LDPF by $t$.

The following proposition summarizes some of the basic properties of $U_{\mathrm{LD}}(s, t)$. They are related to basic properties of free energy.

Proposition 1 The LDPF $U_{\mathrm{LD}}(s, t)$ defined in Equation (1) satisfies the following:

(i) If the process $\{X(t)\}$ has independent increments, then $U_{\mathrm{LD}}(s, t)$ does not depend on $t$.

(ii) If $X(t)=\sum_{i=1}^{n} X_{i}(t)$, where $\left\{X_{i}(t), i=1,2, \ldots, n\right\}$ form a sequence of probabilistically independent financial objects, then the LDPF for $X(t)$ is the sum of the LDPFs for the $X_{i}(t)$.

(iii) For fixed $t, U_{\mathrm{LD}}(s, t)$ is decreasing in $s \geq 0$ and

$$
\frac{\underline{X}(t)}{t} \leq U_{\mathrm{LD}}(s, t) \leq \frac{E(X(t))}{t}
$$

where $\underline{X}(t)=\inf \{x: P(X(t)<x)>0\}$ is the essential infimum of random variable $X(t)$, possibly equal to $-\infty$.

(iv) If $U_{\mathrm{LD}}(s, t)<\infty$ for some $s>0$, then as $s \rightarrow 0$

$$
U_{\mathrm{LD}}(s, t)=\frac{E(X(t))}{t}-\frac{s}{2 t} \operatorname{Var}(X(t))+o(s) .
$$

Proof: The first point follows as $E(\exp (-s X(t)))=E(\exp (-s X(1)))^{t}$ if $X(t)$ has independent increments. The second follows as $E(\exp (-s X(t)))=E\left(\exp \left(-s \sum_{i=1}^{n} X_{i}(t)\right)\right)=$ $\prod_{i=1}^{n} E\left(\exp \left(-s X_{i}(t)\right)\right)$ if the sequence $\left\{X_{i}(t)\right\}$ is formed of independent random variables. The monotonicity in the third point follows immediately from the definition, while the left hand bound follows as $\exp (-s \underline{X}(t)) \geq E(\exp (-s X(t)))$ and the right hand bound follows from Jensen's inequality: $E(\exp (-s X(t))) \geq \exp (-s E(X(t)))$. The final point follows from a Taylor expansion around $s=0$. 
The function $U_{\mathrm{LD}}$ defined in Equation (1) can be motivated in a number of ways. For example, its form is similar to a cumulant generating function. This introduces the desirable properties (i), (ii) for independent financial objects in Proposition 1. Most importantly, it enters naturally into the bounds of the following section.

\section{Using LDPFs to choose a portfolio}

Assume throughout that at time 0 the investor has initial capital $K$ and the ability to invest in $J \geq 1$ distinct probabilistically independent financial objects. For each $t \geq 0$, let $X_{j}(t)$ denote the value of return for the financial object $j \in\{1, \ldots, J\}$ at time $t$. Let $U_{\mathrm{LD}}^{(j)}(s, t)$ denote the LDPF for $X_{j}$. The initial capital constraint ensures that there exists portfolio weights $c_{j} \geq 0, j \in\{1, \ldots, J\}$, such that $K=c_{1} X_{1}(0)+c_{2} X_{2}(0)+\ldots+c_{J} X_{J}(0)$. Let $X(t)$ be the value of the portfolio at time $t$ :

$$
X(t)=c_{1} X_{1}(t)+\ldots+c_{J} X_{J}(t) .
$$

Next, fix the value of an unacceptable loss $-V$, where $V \in(0, \infty)$, and introduce $\gamma>0$, a positive number such that the investor wishes to keep the probability of unacceptable loss below $\exp (-\gamma)$.

Owing to independence, the LDPF for a portfolio $X(t)$ is related to the LDPFs for its components $X_{1}(t), \ldots, X_{J}(t)$ by:

$$
U_{\mathrm{LD}}(s, t)=\frac{-1}{s t} \log E\left(\exp \left[-s \sum_{j=1}^{J} c_{j} X_{j}(t)\right]\right)=\sum_{j=1}^{J} c_{j} U_{\mathrm{LD}}^{(j)}\left(c_{j} s, t\right) .
$$

The investor wishes to identify weights $\left\{c_{j}, j \in\{1, \ldots, J\}\right\}$ that maximize $E(X(t))$ subject to one of the following conditions:

1. the probability of unacceptable loss at the trading time scale $t$ is bounded above by $\exp (-\gamma), P(X(t) \leq-V) \leq \exp (-\gamma)$

2. the probability the portfolio's value is less than an unacceptable loss at a finite collection of times, $\left\{t_{1}, \ldots, t_{M}\right\}$ where $t_{1}<t_{2}<\ldots<t_{M}=t$, prior to and including the trading time scale $t$ is bounded above by $\exp (-\gamma), P\left(X\left(t^{*}\right) \leq-V\right.$, for all $\left.t^{*} \in\left\{t_{1}, \ldots, t_{M}\right\}\right) \leq \exp (-\gamma)$;

3. the probability the portfolio's value is less than an unacceptable loss at any time in the future is bounded above by $\exp (-\gamma), P\left(X(t) \leq-V\right.$, for all $\left.t \in \mathbb{R}_{+}\right) \leq \exp (-\gamma)$.

We give explicit bounds for the first two constraints in terms of the LDPFs of the financial objects. For the third constraint, we employ a large deviation limit theorem using the LDPF. 
The bounds enable an identification of portfolios that satisfy these constraints. Of these portfolios, the investor then selects the one that maximizes the expected return.

A sufficient condition for a portfolio to satisfy constraint 1 is given by Chernoff's bound:

$$
P(X(t) \leq-V) \leq \exp \left(\inf _{s}\left[-s\left(t U_{\mathrm{LD}}(s, t)+V\right)\right]\right) .
$$

Then a range of acceptable portfolios satisfying constraint 1 is

$$
A_{1}(\gamma, V)=\left\{\left(c_{1}, \ldots, c_{J}\right) \in \mathbb{R}_{+}^{J}: \inf _{s}\left[-s\left(\sum_{j=1}^{J} c_{j} t U_{\mathrm{LD}}^{(j)}\left(c_{j} s, t\right)+V\right)\right] \leq-\gamma\right\} .
$$

That is, a portfolio constructed of any $\left(c_{1}, \ldots, c_{J}\right) \in A_{1}(\gamma, V)$ will meet our investor's constraint. Thus his objective in this case is to maximize

$$
\frac{E(X(t))}{t}=\sum_{j=1}^{J} c_{j} U_{\mathrm{LD}}^{(j)}(0, t) \text { subject to }\left(c_{1}, \ldots, c_{J}\right) \in A_{1}(\gamma, V)
$$

A sufficient condition for a portfolio to satisfy constraint 2 is again given by a union bound followed by Chernoff's bound:

$$
P\left(\bigcup_{i=1}^{M} X\left(t_{i}\right) \leq-V\right) \leq \sum_{i=1}^{M} P\left(X\left(t_{i}\right) \leq-V\right) \leq \sum_{i=1}^{M} \exp \left(\inf _{s}\left[-s\left(t_{i} U_{\mathrm{LD}}\left(s, t_{i}\right)+V\right)\right]\right) .
$$

This bound is loose unless the value $V>0$ is large, but becomes tight as $V$ increases. By the principle of the largest term, when $V$ is large then only a single, most volatile time dominates. A range of acceptable portfolios satisfying constraint 2 is specified by

$$
A_{2}(\gamma, V)=\left\{\left(c_{1}, \ldots, c_{J}\right) \in \mathbb{R}_{+}^{J}: \sum_{i=1}^{M} \exp \left(\inf _{s}\left[-s\left(\sum_{j=1}^{J} c_{j} t_{i} U_{\mathrm{LD}}^{(j)}\left(c_{j} s, t_{i}\right)+V\right)\right]\right) \leq e^{-\gamma}\right\} .
$$

As one would expect, the second constraint is more restrictive than the first so that $A_{2}(\gamma, V) \subset$ $A_{1}(\gamma, V)$. Thus the investor's objective in this case is to maximize

$$
\sum_{j=1}^{J} c_{j} U_{\mathrm{LD}}^{(j)}(0, t) \text { subject to }\left(c_{1}, \ldots, c_{J}\right) \in A_{2}(\gamma, V)
$$

Identifying a sufficient condition for a portfolio to satisfy constraint 3 is significantly more challenging. Dealing with an infinite time horizon introduces non-trivial mathematical difficulties. For return processes that do not possess long-range dependencies, we adopt an 
approximation based on large deviation theory taken from Ganesh and O'Connell [6]. Processes with long-range dependencies must be treated on a case-by-case basis through, for example, the theory in Duffy, Lewis and Sullivan [7].

Although this approach restricts the result to large values of $V$ (it would be preferable to obtain a strict bound for all $V$ ), there is an additional benefit of the techniques used in [6]: this techniques predicts both the "most likely" path to an unacceptable loss and the most likely time scale for an unacceptable loss.

We start by noting the following equivalence for the value of a given portfolio $X(t)$ :

$$
P\left(X(t) \leq-V \text {, any } t \in \mathbb{R}_{+}\right)=P\left(\inf _{t \in \mathbb{R}_{+}} X(t) \leq-V\right) .
$$

Next, define the following function for $s \in[0, \infty]$ :

$$
\chi(s)=\lim _{t \rightarrow \infty}-s U_{\mathrm{LD}}(s, t),
$$

assuming that the limit exists in $[-\infty, \infty]$. Furthermore, suppose that

$$
\chi^{\prime}(0)=\left.\frac{d}{d s} \chi(s)\right|_{s=0}=-\mu=\lim _{t \rightarrow \infty}-\frac{E(X(t))}{t}<0 .
$$

Note that if $X(t)=\sum_{j=1}^{J} c_{j} X_{j}(t)$ and the processes $X_{1}(t), \ldots, X_{J}(t)$ are independent, then

$$
\chi(s)=-\sum_{j=1}^{J} c_{j} s \lim _{t \rightarrow \infty} U_{\mathrm{LD}}^{(j)}\left(c_{j} s, t\right),
$$

where $U_{\mathrm{LD}}^{(j)}$ is the LDPF for $\left\{X_{j}(t)\right\}$.

Should a range of technical conditions in [6] and [8] on $\{X(t)\}$ be satisfied (which is the case in our examples, apart from Fractional Brownian Motion), then

$$
\lim _{V \rightarrow \infty} \frac{1}{V} \log P\left(\inf _{t \in \mathbb{R}_{+}} X(t) \leq-V\right)=-\sup \{s \geq 0: \chi(s) \leq 0\}=:-\delta,
$$

so that

$$
P\left(\inf _{t \in \mathbb{R}_{+}} X(t) \leq-V\right) \approx \exp (-V \delta) .
$$

Then a range of acceptable portfolios satisfying constraint 3 for large $V$ is

$$
A_{3}(\gamma, V)=\left\{\left(c_{1}, \ldots, c_{J}\right) \in \mathbb{R}_{+}^{J}:-V \sup \left\{s \geq 0:-\sum_{j=1}^{J} \lim _{t \rightarrow \infty} c_{j} U_{\mathrm{LD}}^{(j)}\left(c_{j} s, t\right) \leq 0\right\} \leq-\gamma\right\} .
$$


That is, a portfolio constructed of any $\left(c_{1}, \ldots, c_{J}\right) \in A_{3}(\gamma, V)$ will meet our investor's constraint if $V$ is large. Thus his objective in this case is to maximize

$$
\lim _{t \rightarrow \infty} \sum_{j=1}^{J} c_{j} U_{\mathrm{LD}}^{(j)}(0, t) \text { subject to }\left(c_{1}, \ldots, c_{J}\right) \in A_{3}(\gamma, V) .
$$

Moreover, the theory tells us that if $\chi^{\prime}(\delta)$ is finite, as $V \rightarrow \infty$ the most likely time for an unacceptable loss is $V / \chi^{\prime}(\delta)$ and the most likely path to an unacceptable loss is approximately piecewise linear:

$$
X(t) \approx \begin{cases}-t \chi^{\prime}(\delta) & \text { for } t \leq \frac{V}{\chi^{\prime}(\delta)} \\ -V+\mu\left(t-\frac{V}{\chi^{\prime}(\delta)}\right) & \text { for } t \geq \frac{V}{\chi^{\prime}(\delta)} .\end{cases}
$$

Examples of these results will be given later.

\section{Example LDPFs}

\subsection{Gaussian return processes}

Consider the process $\left\{X(t), t \in \mathbb{R}_{+}\right\}$defined by $X(t)=\mu t+Z(t)$, with positive drift $\mu>0$, where $Z(t)$ is Gaussian with zero mean. Define the covariance function $R\left(t_{1}, t_{2}\right)=$ $E\left[Z\left(t_{1}\right) Z\left(t_{2}\right)\right]$, so that $\sigma_{t}^{2}=R(t, t)$. The LDPF is given by

$$
U_{\mathrm{LD}}(s, t)=\mu-\frac{s}{2 t} \sigma_{t}^{2} .
$$

A commonly used Gaussian model that possesses both self-similarity and long-range dependence is Fractional Brownian Motion (FBM). It was first introduced by Kolmogorov [9] under the name Wiener spiral to model turbulence in fluid flows and later introduced into finance by Mandelbrot and Van Ness [10] as a modeling tool suitable for explaining self-similar time series. Its paths are almost surely continuous functions. Detailed discussion on properties of FBM can be found in Samorodnitsky and Taqqu [11]. FBM is a Gaussian process with stationary increments whose covariance function takes the following form

$$
2 R(t, s)=t^{2 H}+s^{2 H}-|t-s|^{2 H}, \quad \sigma_{t}^{2}=\sigma^{2} t^{2 H}, \quad H \in(0,1),
$$

where $H$ is called the Hurst parameter. When $H=0.5$ we get standard Brownian Motion, also known as the Wiener-Bachelier process, which is short-range dependent and essentially corresponds to the classical Markowitz setting. Equation (5) gives

$$
U_{\mathrm{LD}}(s, t)=\mu-\frac{s \sigma^{2}}{2} t^{2 H-1}
$$

for the LDPF of FBM. 


\section{$4.2 \quad$ Jump diffusion return processes}

The Gaussian model of the previous section gives continuous sample paths, almost surely. Merton [12] introduced the Jump Diffusion (JD) model of stock returns which possesses properties observed in practice: negative skewness and excess kurtosis of the probability density of log returns. It is constructed as the sum of two independent processes, a continuous diffusion and a discontinuous jump process, and its paths are almost surely discontinuous.

The continuous diffusion is Brownian Motion with drift $\mu>0$ corresponding to $X(t)$ with $H=$ $0.5, \sigma^{2}=1$ in Section 4.1 and denoted $B(t)$. The discontinuous jump process is a compound Poisson process, $\sum_{i=1}^{N(t)} Y_{i}$, where the sequence $\left\{Y_{i}\right\}$ is independent and identically distributed with $Y_{1}$ having the normal distribution and $\{N(t), t \geq 0\}$ is a standard Poisson process with rate $\eta$. Thus the jump process has two elements of randomness: random summands and a random number of summands. Define

$$
X(t)=\mu t+B(t)+\sum_{i=1}^{N(t)} Y_{i}
$$

which models the log return process so that the real return process is given by $S(t)=$ $S(0) \exp (X(t))$. We can bound $P\left(S(t) \leq V^{\prime}\right)$ be using the standard bounds on $P(X(t) \leq-V)$ and setting $V^{\prime}=S(0) \exp (-V)$, so we focus on calculating LDPF for $X(t)$.

Using property 2 of Proposition 1, calculating $U_{\mathrm{LD}}$ for $X(t)$ is straight forward. We know that $U_{\mathrm{LD}}$ for $\mu t+B(t)$ is given by Equation (6) with $H=0.5: \mu-s / 2$. Moreover it is well known that

$$
\frac{1}{t} \log E\left(\exp \left(s \sum_{i=1}^{N(t)} Y_{i}\right)\right)=\eta\left[\exp \left(\frac{s^{2}}{2}\right)-1\right],
$$

so that the $\mathrm{LDPF}$ for $\sum_{i=1}^{N(t)} Y_{i}$ is $-s^{-1} \eta\left(\exp \left(s^{2} / 2\right)-1\right)$. Thus for all $s \in \mathbb{R}$

$$
U_{\mathrm{LD}}(s, t)=\mu-\frac{s}{2}-\frac{\eta}{s}\left[\exp \left(\frac{s^{2}}{2}\right)-1\right] .
$$

\subsection{Variance Gamma return processes}

This model, its history and many of its properties are described in detail by Madan, Carr and Chang [13]. A Variance Gamma (VG) process is defined by subordinating a Brownian Motion with drift by a gamma process with unit rate (see, for example, Feller [14]). Let $\{g(t), t \in \mathbb{R}\}$ denote a gamma process with unit rate; that is, a process with independent increments, whose increments over a length $h$ are distributed by a gamma distribution with mean $h$ and variance $\nu h, \nu>0$. Let $\sigma B(t)$ denote a Brownian Motion, where $\sigma$ denotes its volatility, and let it have drift $\mu>0$, then the VG model of returns is given by

$$
X(t)=\mu(g(t))+\sigma B(g(t)) .
$$


That is, $X(t)$ is Brownian Motion with drift evaluated at random time determined by the gamma process. Again, $X(t)$ is often treated as the log return process, so that the return process is given by $S(t)=S(0) \exp (X(t))$.

Due to the nature of the gamma process, the VG process has infinitely many small jumps and a finite number of large jumps. The introduction of the extra parameter to the Brownian Motion by the subordination enables the VG model to capture the negative skewness and excess kurtosis of the log returns which is often reported in empirical investigation. The variance parameter $\nu$ of the gamma process controls the degree of randomness of subordination. Large values of $\nu$ result in fatter tails of the log return density. The drift parameter of the subordinated Brownian Motion process $\mu$ captures skewness of the log returns.

Equation (7) of [13] gives an explicit expression for the characteristic function, of $X(t)$

$$
\phi_{X(t)}(s)=E\left(\exp (i s X(t))=\left(\frac{1}{1-\mu \nu i s+\left(\sigma^{2} \nu / 2\right) s^{2}}\right)^{t / \nu}, s \in \mathbb{R} .\right.
$$

The function $\phi_{X(t)}(s)$ can be analytically continued, having poles at

$$
s=i\left(\mu \nu+\sqrt{\mu^{2} \nu^{2}+2 \sigma^{2} \nu}\right) /\left(\sigma^{2} \nu\right) \text { and } s=i\left(\mu \nu-\sqrt{\mu^{2} \nu^{2}+2 \sigma^{2} \nu}\right) /\left(\sigma^{2} \nu\right),
$$

which, as $\nu>0$, are positive and negative respectively. Thus the moment generating function $M_{X(t)}(s)=E\left(\exp (s X(t))=\phi_{X(t)}(-i s)\right.$ for $s$ in a neighborhood of the origin and the LDPF for $X(t)$ is given by:

$$
\begin{aligned}
U_{\mathrm{LD}}(s, t)= & -\frac{1}{s t} \log \phi_{X(t)}(i s)=\frac{1}{s \nu} \log \left(1+\mu \nu s-\frac{\sigma^{2} \nu}{2} s^{2}\right), \\
& s \in\left(\frac{\mu \nu-\sqrt{\mu^{2} \nu^{2}+2 \sigma^{2} \nu}}{\sigma^{2} \nu}, \frac{\mu \nu+\sqrt{\mu^{2} \nu^{2}+2 \sigma^{2} \nu}}{\sigma^{2} \nu}\right) .
\end{aligned}
$$

Note that the LDPF in Equation (7) can be rewritten as

$$
\frac{1}{s \nu} \log \left(1+s \nu U_{\mathrm{LD}}^{B M}(s, t)\right)
$$

where $U_{\mathrm{LD}}^{B M}$ is the LDPF for Brownian Motion, so that for small $s$ we have $U_{\mathrm{LD}}(s, t) \approx$ $U_{\mathrm{LD}}^{B M}(s, t)$.

\subsection{Truncated Lévy return processes}

For certain financial objects it has been suggested that while on short time scales fluctuations in value are large, in the longer term they are not. This has led to the proposal of the truncated Lévy (TL) process as a model for the dynamics of mature financial markets. For an overview see Mantegna and Stanley [15]. The TL process can explain large fluctuations in value at 
short time scales, as well as convergence to a Gaussian process at longer time scales. Here, as suggested by Matacz [16], we focus on symmetric distributions.

Let $X$ be a random variable with a symmetric Lévy distribution. The distribution is most conveniently defined by the characteristic function of $X$

$$
\phi_{X(t)}(s)=E\left(\exp (i s X(t))=\exp \left(-c^{\alpha}|s|^{\alpha}\right)\right.
$$

where $c$ is a scale parameter and $\alpha \in(0,2]$. This characteristic function is not explicitly invertible unless $\alpha=2$, which gives a Gaussian, or $\alpha=1$, which gives a Cauchy distribution. When $\alpha<2$, the Lévy distribution has infinite variance. When $\alpha \leq 1$, it also has infinite mean. A Lévy process is a process $\left\{S_{n}, n \in \mathbb{N}\right\}$ of partial sums $S_{n}=X_{1}+\cdots+X_{n}$, where $\left\{X_{n}, n \in \mathbb{N}\right\}$ are independent and identically distributed (i.i.d.) with a Lévy distribution. Thus when $\alpha<2$, the central limit theorem does not apply to $\left\{S_{n}, n \in \mathbb{N}\right\}$.

In contrast to the Lévy distribution, originally suggested by Mandelbrot as a useful model for financial objects, the TL distribution has all moments finite. With the TL process defined as a partial sum process of i.i.d. random variables, each with the TL distribution, one can account for both observed excess kurtosis at short time scales and a slow convergence to a Gaussian at longer time scales.

The TL distribution is defined so that it behaves as a Lévy distribution in the central part of its support, but has tails that decay no slower than exponentially. This ensures that the variance of the TL distribution is finite. Therefore the TL process satisfies the central limit theorem. Depending on the point at which the Lévy behavior no longer dominates, there emerges a characteristic time scale which separates the Lévy and Gaussian regimes. This time scale can be made arbitrary long. In [15], the tail of the Lévy distribution is truncated by setting it to zero beyond a threshold. Here we adopt the strategy from Koponen [17] where the truncation arises as an exponential decay with rate $\beta$.

Restricting Equation (3) of [17] to symmetric distributions gives the following characteristic function for a random variable $X$ distributed with a TLD:

$$
\phi_{X}(s)=\exp \left(-\frac{c^{\alpha}}{\cos (\pi \alpha / 2)}\left(\left(s^{2}+\beta^{2}\right)^{\alpha / 2} \cos (\alpha \arctan (s / \beta))-\beta^{\alpha}\right)\right), s \in \mathbb{R},
$$

so long as $\alpha \neq 1,2$. This characteristic function can be analytically continued with poles at $s= \pm i \beta$, so that the moment generating function $M_{X}(s)=E(\exp (s X))=\phi_{X}(-i s)$ for $s \in(-\beta, \beta)$. This is as we would expect, as the distribution is truncated by a two-sided exponential. We define for any $n \in \mathbb{N}, X(n)=X_{1}+\ldots+X_{n}$ where $\left\{X_{t}, t \in \mathbb{N}\right\}$ are i.i.d distributed as a TLD. As, for any $z \in \mathbb{C}, \cos (z)=(\exp (i z)+\exp (-i z)) / 2$ and, for any $z \in \mathbb{C}$ such that $|z|<1, \arctan (z)=(\log (1-i z)-\log (1+i z)) i / 2$, the LDPF for $X(n)$ is

$U_{\mathrm{LD}}(s, n)=\frac{c^{\alpha}}{s \cos (\pi \alpha / 2)}\left(\left(\beta^{2}-s^{2}\right)^{\alpha / 2} \frac{\left(\left(1-\frac{s}{\beta}\right)^{\frac{\alpha}{2}}\left(1+\frac{s}{\beta}\right)^{\frac{-\alpha}{2}}+\left(1-\frac{s}{\beta}\right)^{\frac{-\alpha}{2}}\left(1+\frac{s}{\beta}\right)^{\frac{\alpha}{2}}\right)}{2}-\beta^{\alpha}\right)$, 
for $n \in \mathbb{N}, \alpha \in(0,2)$, and $s \in(-\beta, \beta)$.

\subsection{Summary}

For the FBM process, $\mu$ is the drift, $\sigma^{2} \geq 0$ the variance and $H \in(0,1)$ is the Hurst parameter. For the JD process, $\mu$ is again the drift, while $\eta>0$ is the rate of the Poisson process of jumps. For the VG process, $\mu$ is the drift and $\sigma$ the volatility of the underlying Brownian Motion, while $\nu>0$ is the variance rate of the Gamma process (which is assumed to have mean rate 1$)$. For the TL process, $c$ is a scale constant, $\alpha \in(0,2), \alpha \neq 1$, is the Lévy parameter and $\beta>0$ is the exponential cut-off parameter.

\begin{tabular}{|c|c|}
\hline$(\log )$ Return Process & $U_{\mathrm{LD}}(s, t)$ \\
\hline \hline $\mathrm{FBM}\left(\mu, \sigma^{2}, H\right)$ & $\mu-s \sigma^{2} t^{2 H-1} / 2$ \\
$(\log ) \mathrm{JD}(\mu, \eta)$ & $\mu-s / 2-\left(\exp \left(s^{2} / 2\right)-1\right) \eta / s$ \\
$(\log ) \mathrm{VG}(\mu, \nu, \sigma)$ & $\log \left(1+\mu \nu s-\sigma^{2} \nu s^{2} / 2\right) /(s \nu)$ \\
$(\log ) \mathrm{TL}(c, \alpha, \beta)$ & $\frac{c^{\alpha}}{s \cos (\pi \alpha / 2)}\left[\left(\beta^{2}-s^{2}\right)^{\alpha / 2}\left(k^{\alpha / 2} \widehat{k}^{-\alpha / 2}+k^{-\alpha / 2} \widehat{k}^{\alpha / 2}\right) / 2-\beta^{\alpha}\right]$ \\
& where $k=1-s / \beta$ and $\widehat{k}=1+s / \beta$ \\
\hline
\end{tabular}

For the FBM and the JD processes, the variable $s$ is allowed to vary in the whole of $\mathbb{R}$. For the VG process, $U_{\mathrm{LD}}$ is infinite for $s$ outside of the range defined in (7). For the TL process, $U_{\mathrm{LD}}$ is infinite for $s \notin(-\beta, \beta)$.

\section{Portfolio examples: finite trading time scale}

\subsection{Single unit of Fractional Brownian Motion returns}

A calculation of the Chernoff bound in Equation (3) corresponding to constraint 1 reveals for a portfolio consisting of single unit of this financial object at trading time $t$

$$
P(X(t) \leq-V) \leq \exp \left(-\frac{(\mu t+V)^{2}}{2 \sigma^{2} t^{2 H}}\right)
$$

One can then turn this around and ask: given a probabilistic tolerance $\gamma$, what value must $V(t)$ take to ensure that $P(X(t) \leq-V(t)) \leq \exp (-\gamma)$ ? The Chernoff bound answers this question with the inequality

$$
V(t) \geq t^{H} \sqrt{2 \sigma^{2} \gamma}-t \mu .
$$

That is, for given $\gamma$ and $t$, a single unit of this financial object will satisfy constraint 1 if the unacceptable loss is bounded as in (8). From (8) one can identify the most volatile trading time. That is, the trading time $t^{*}$ at which the lower bound on $V\left(t^{*}\right)$ is greatest (the 


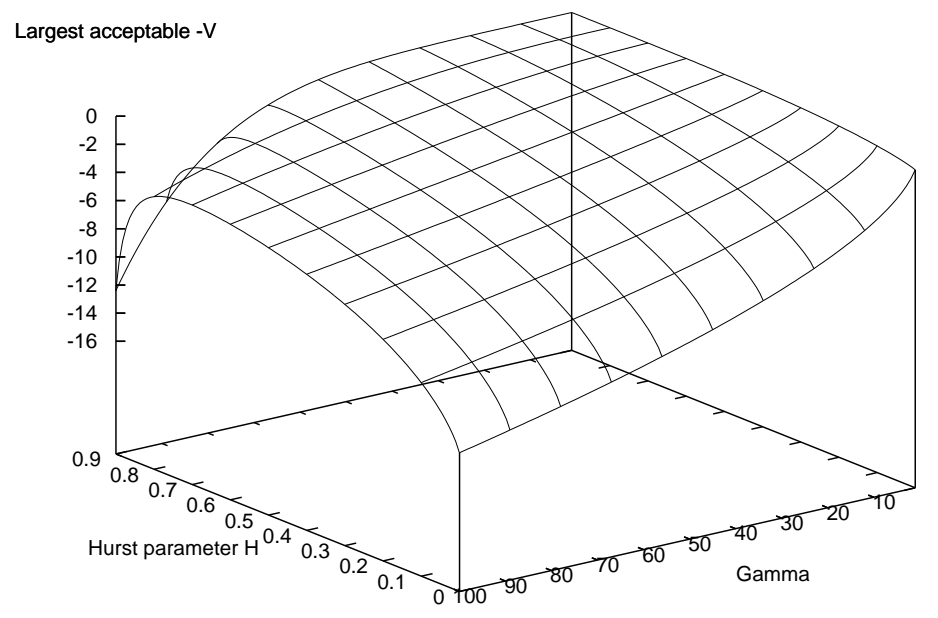

Figure 1: FBM with $\sigma^{2}=1$ and $\mu=10$. Plot of maximum acceptable loss determined by the Chernoff bound at the most volatile trading time as a function of $\gamma$ and $H$.

unacceptable loss is highest) by maximizing the right hand side of (8) with respect to time, which gives

$$
t^{*}=\left(\frac{H \sqrt{2 \sigma^{2} \gamma}}{\mu}\right)^{1 /(1-H)}
$$

corresponding to a minimum unacceptable loss of

$$
\left(2 \sigma^{2} \gamma\right)^{1 /(2(1-H))}\left(\frac{H}{\mu}\right)^{1 /(1-H)} \mu\left(\frac{1}{H}-1\right) .
$$

For given $\mu$ and $\sigma^{2}$, Figure 1 plots the highest value of $-V$ that satisfies the most volatile time bound for a range of $\gamma$ and $H$. As one would expect, there is a strong dependence on the Hurst parameter $H$.

\subsection{Single unit of Jump Diffusion returns}

Consider a portfolio consisting of a single unit of stock whose stochastic nature is that of a JD process. The point at which $\inf _{s}\left(-s t U_{\mathrm{LD}}(s, t)-s V\right)$ is obtained is found by differentiating the argument with respect to $s$ and setting it equal to zero. This gives a transcendental equation that cannot be solved explicitly, but can be solved readily by computer. For example, Figure 2 plots $-\inf _{s}\left(-s t U_{\mathrm{LD}}(s, t)-s V\right)$. To put it another way, for given $V$, Figure 2 plots the 


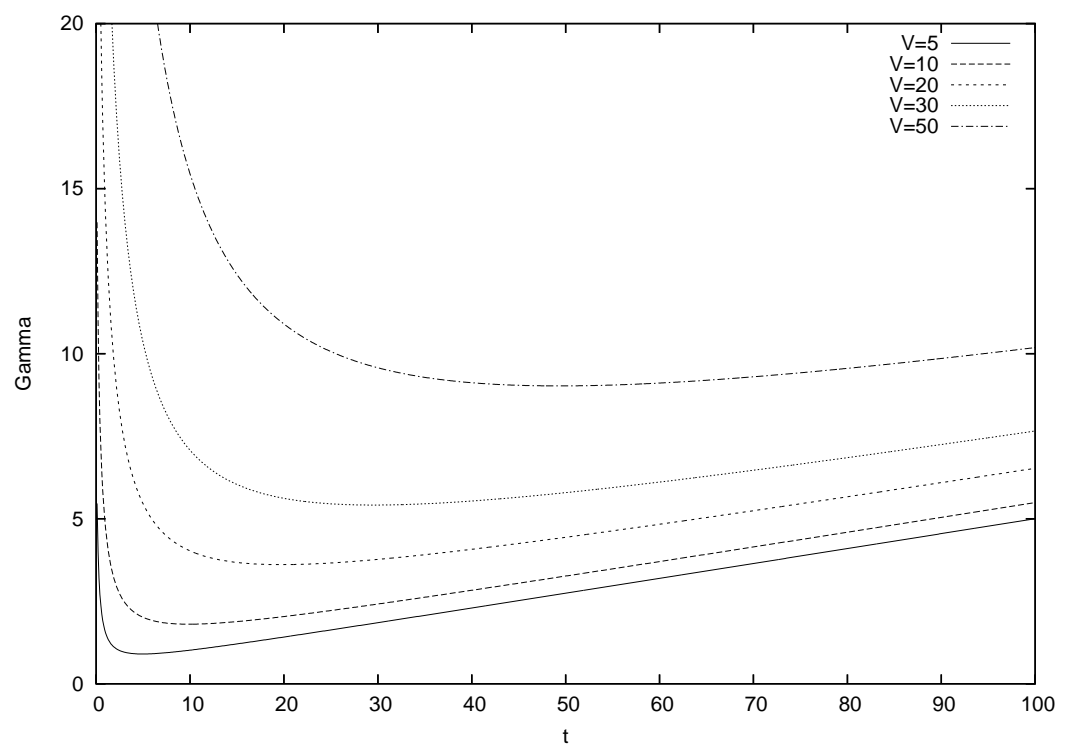

Figure 2: Jump Diffusion process with $\mu=1$ and $\eta=10$. Plot of $\gamma(t)$ against $t$, for a range of $V$.

smallest $\gamma(t)$ such that the Chernoff bound says $P(S(t) \leq-V) \leq \exp (-\gamma(t))$. Note the emergence of a most volatile time for given $V$.

\subsection{Single unit of Variance Gamma returns}

Consider a portfolio consisting of a single unit of stock whose stochastic nature is that of a Variance Gamma process. The positive argument at which $\inf _{s}\left(-s t U_{\mathrm{LD}}(s, t)-s V\right)$ is obtained can be determined explicitly to be

$$
\frac{\mu \nu V-t \sigma^{2}+\sqrt{t^{2}\left(\sigma^{2}\right)^{2}+\mu^{2} \nu^{2} V^{2}+2 \sigma^{2} \nu V^{2}}}{\sigma^{2} \nu V} .
$$

For example Figure 3 plots $-\inf _{s}\left(-s t U_{\mathrm{LD}}(s, t)-s V\right)$. Again note the emergence of a volatile trading time for given $V$.

\subsection{Single unit of Truncated Lévy returns}

Consider a portfolio consisting of a single unit of stock whose stochastic nature is that of a TL process, but with drift $\mu$, so that $X(t)=\mu(t)+L(t)$ for $t \in \mathbb{N}$, where $L(t)$ is a truncated Lévy return. The argument at which $\inf _{s}\left(-s t U_{\mathrm{LD}}(s, t)-s V\right)$ is obtained is found by differentiating 


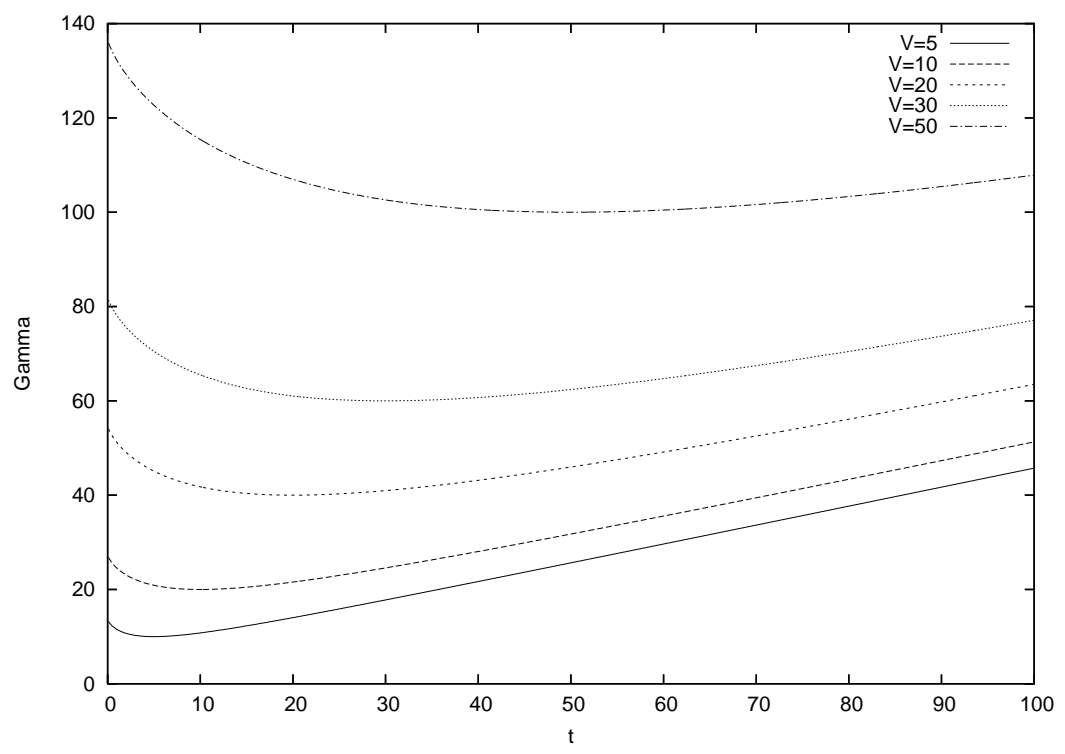

Figure 3: Variance Gamma process with $\mu=1, \nu=1$ and $\sigma^{2}=1$. Plot of $\gamma(t)$ against $t$, for a range of $V$.

the argument with respect to $s$ and setting it equal to zero. This gives an equation can be solved readily by computer. For example Figure 2 plots $-\inf _{s}\left(-s t U_{\mathrm{LD}}(s, t)-s V\right)$.

\subsection{Tradeoff between a risk-free asset and FBM returns}

Consider an investor that can divide an initial investment between a risky asset whose statistics are governed by FBM and a non-risky asset. The non-risky asset's value at time $t$ is $\mu_{1} t$, while the FBM has parameters $\left(\mu_{2}, \sigma^{2}, H\right)$, where $\mu_{2}$ is assumed greater than $\mu_{1}$. If the proportion of initial capital invested in the non-risky asset is $1-c$ and the proportion invested in the risky asset is $c$, then the LDPF for the portfolio is given by (2):

$$
U_{\mathrm{LD}}(s, t)=\mu_{1}+c\left(\mu_{2}-\mu_{1}\right)-\frac{c^{2} s \sigma^{2} t^{2 H-1}}{2} .
$$

This corresponds to FBM with $\left(\mu_{1}+c\left(\mu_{2}-\mu_{1}\right), c^{2} \sigma^{2}, H\right)$. Thus for trading time scale $t$ and unacceptable loss $-V$ the Chernoff bound gives

$$
P(X(t) \leq-V) \leq \exp \left(-\frac{\left(\left(\mu_{1} t+c\left(\mu_{2}-\mu_{1}\right)\right) t+V\right)^{2}}{2 c^{2} \sigma^{2} t^{2 H}}\right) .
$$

The investor wishes to decide the split $c$ that maximizes the portfolio's expected at time $t$ subject to $P(X(t) \leq-V) \leq \exp (-\gamma)$. As for given split $c, E(X(t))=\mu_{1}+c\left(\mu_{2}-\mu_{1}\right)$, and 


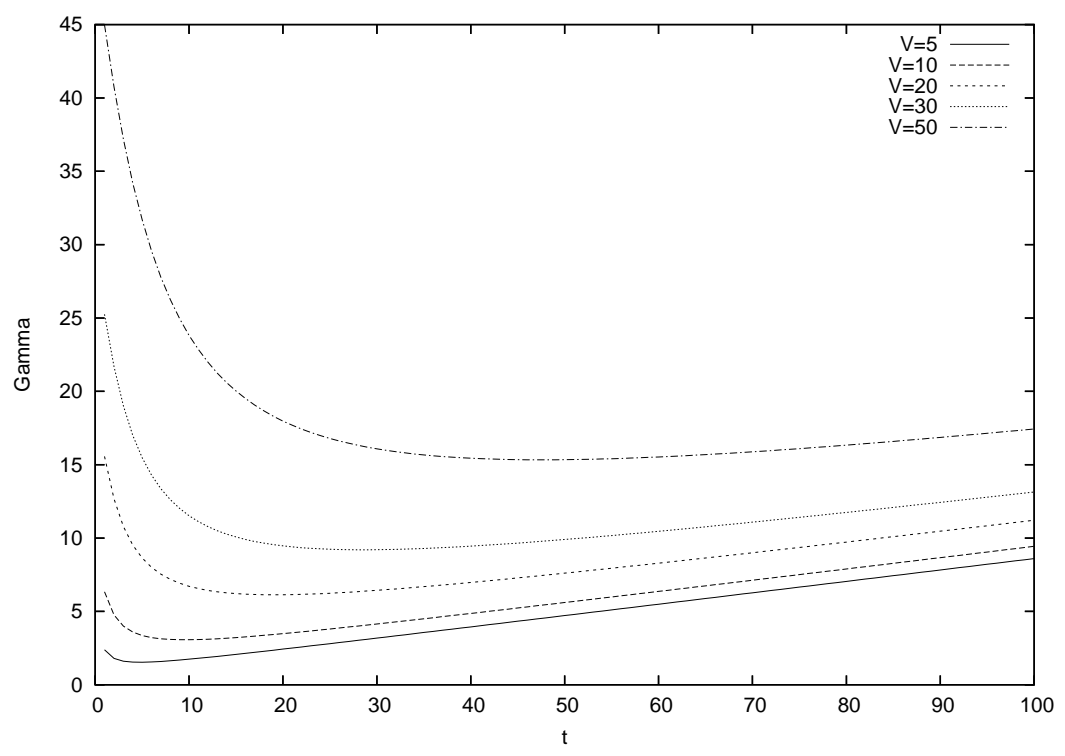

Figure 4: Truncated Lévy process with $c=100, \mu=1, \alpha=0.5$ and $\beta=1$. Plot of $\gamma(t)$ against $t$, for a range of $V$.

$\mu_{2}$ is assumed greater than $\mu_{1}$, he wishes to find

$$
c^{*}=\sup \left\{c \in[0,1]: \frac{\left(\left(\mu_{1}+c\left(\mu_{2}-\mu_{1}\right)\right) t+V\right)^{2}}{2 c^{2} \sigma^{2} t^{2 H}} \geq \gamma\right\} .
$$

Define

$$
t^{*}=\left(\frac{2 \sigma^{2} \gamma}{\left(\mu_{2}-\mu_{1}\right)^{2}}\right)^{\frac{1}{2(1-H)}} .
$$

If $t \geq t^{*}$, then $c^{*}=1$. If $t<t^{*}$, then

$$
c^{*}=\min \left\{\frac{\mu_{1} t+V}{\sqrt{2 \sigma^{2} \gamma} t^{H}-\left(\mu_{2}-\mu_{1}\right) t}, 1\right\} .
$$

Figure 5 plots $c^{*}$ as a function of $t$ for a particular parameterization. If the trading time scale is short or long, $c^{*}$ can be large, but is smaller in an interim regime, demonstrating the importance of the trading time scale. 


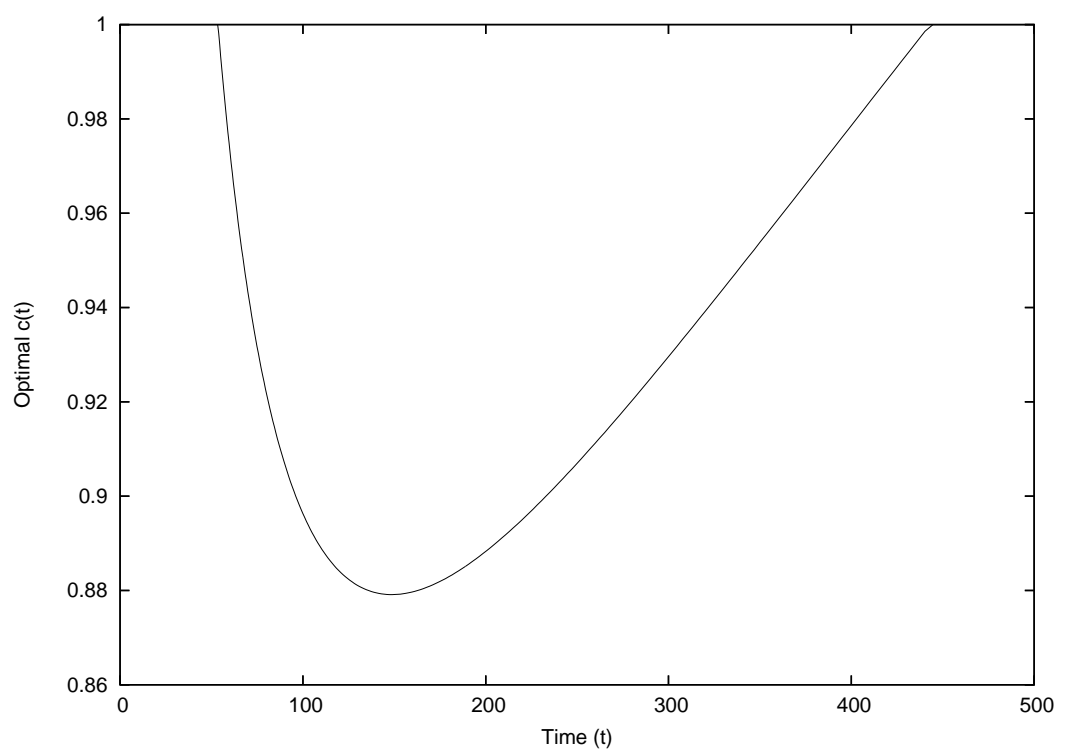

Figure 5: For $\gamma=100$ and $V=100$, optimal split $c^{*}$ for a bond with $\mu_{1}=1$ and stock following FBM with $\sigma^{2}=1, \mu_{2}=1.01$ and $H=0.6$

\section{Portfolio examples: infinite trading time scale}

\subsection{Single unit of Brownian Motion or VG returns}

For a single unit of returns following Brownian Motion using (5) we can readily calculate

$$
\begin{aligned}
\chi(s)=\lim _{t \rightarrow \infty}-s U_{\mathrm{LD}}(s, t) & =\frac{s^{2} \sigma^{2}}{2}-s \mu, \\
\delta=\sup \{s: \chi(s) \leq 0\} & =\frac{2 \mu}{\sigma^{2}} \\
\text { and } \chi^{\prime}(\delta) & =\mu .
\end{aligned}
$$

Thus for large $V$ large deviation theory predicts that

$$
P(X(t) \leq-V, \text { any } t) \approx \exp \left(-V \frac{2 \mu}{\sigma^{2}}\right) .
$$

It also predicts that the most likely time for an unacceptable loss is $V / \mu$. This matches with intuition, the higher the drift $\mu$ is, the less likely an unacceptable loss is to occur at a long time scale. Finally, it predicts that the most likely path to an unacceptable loss is:

$$
X(t) \approx \begin{cases}-t \mu & \text { for } t \leq \frac{V}{\mu} \\ -V+\mu\left(t-\frac{V}{\mu}\right) & \text { for } t \geq \frac{V}{\mu} .\end{cases}
$$




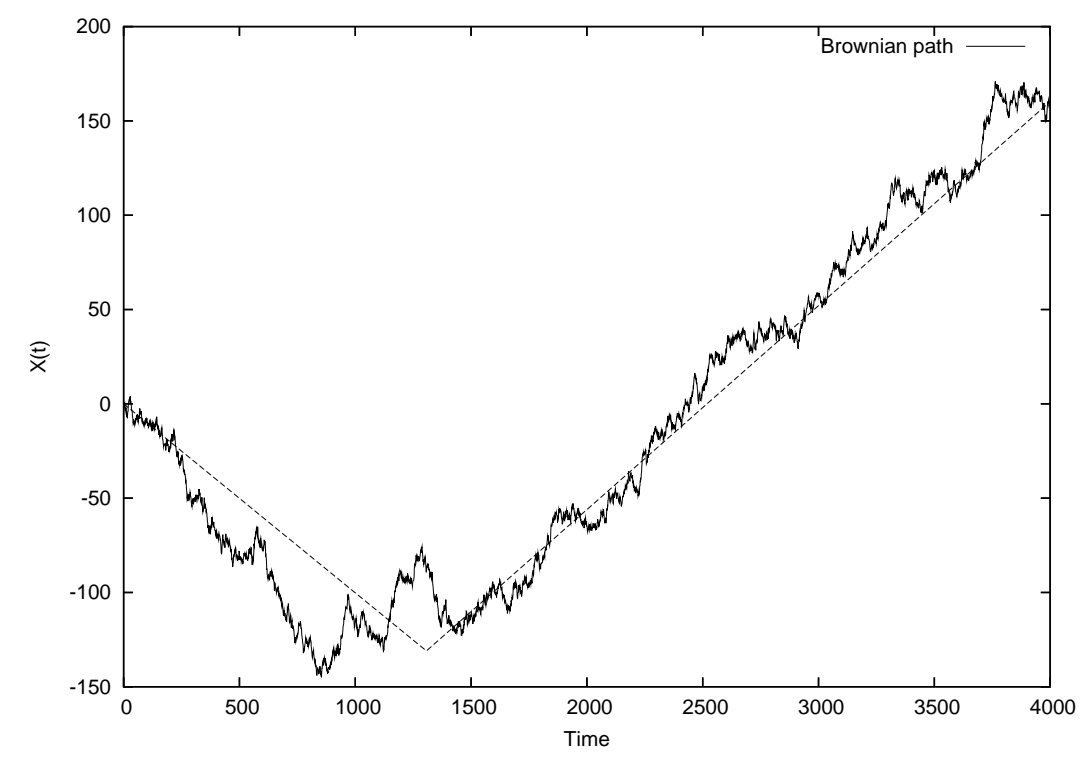

Figure 6: Path of Brownian Motion with $\sigma^{2}=2$ and $\mu=0.1$. Broken line with slope $-\mu$ and then $\mu$ shown for comparison.

To illustrate the path result, a simulation of Brownian Motion with $\mu=0.1$ and $\sigma^{2}=2$ was constructed. Paths of length $t=4000$ were constructed. Figure 6 is the first path observed that dropped below -140. As seeing paths that go below lower levels is exponentially less likely, straight simulation is not feasible. However, as the level is dropped lower, the piecewise linear character of the observed paths becomes more prominent.

Interestingly, for a single unit of VG returns with $U_{\mathrm{LD}}$ given in (7),

$$
\chi(s)=\frac{-1}{\nu} \log \left(1+\mu \nu s-\frac{\sigma^{2} \nu}{2} s^{2}\right)
$$

but all the above statements hold. As the VG process is a time transformed Brownian Motion, subordination cannot increase the density of paths for which an unacceptable loss occurs. Thus, as the subordination has mean drift 1 , an unacceptable loss is most likely to occur at the same time, and in the same way, as Brownian Motion, even though the statistics of the processes are quite different.

\subsection{Single unit of Jump Diffusion or Truncated Lévy returns}

For a portfolio of either JD or TL returns, the function $\chi(s)$ is readily calculable, but determining $\delta$ and $\chi^{\prime}(\delta)$ requires the solution of a transcendental equation. Although this means 


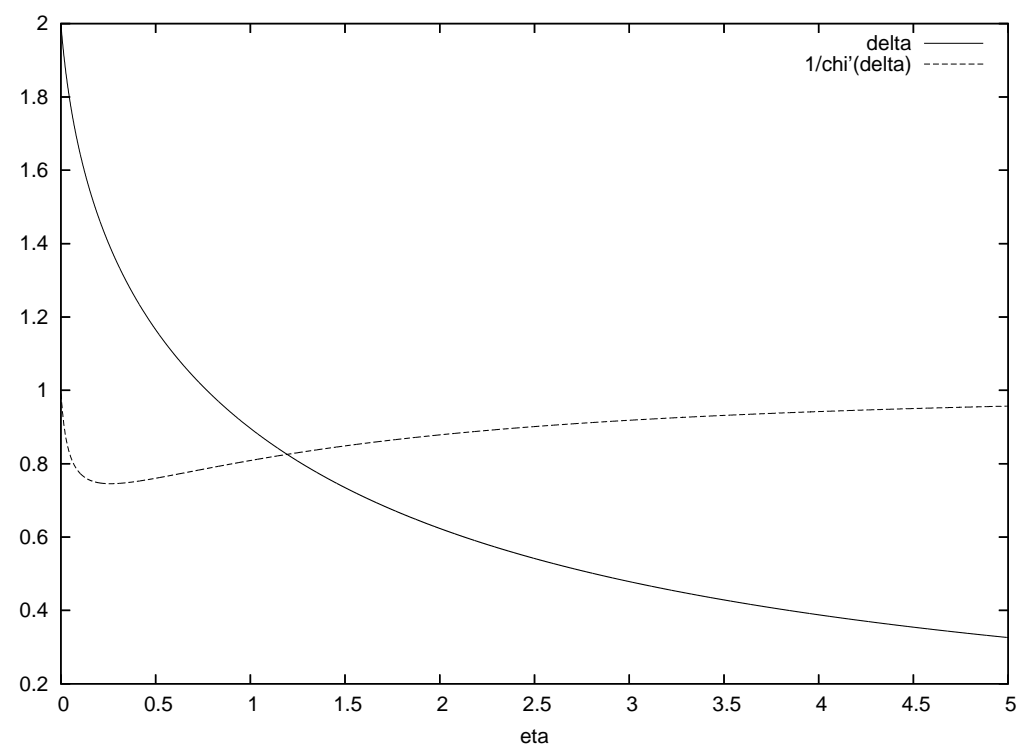

Figure 7: Single unit of JD returns with $\mu=1$. Plot of $\delta$ and $1 / \chi^{\prime}(\delta)$ as a function of $\eta$, the mean arrival rate of jumps

there are no closed-form solutions, in practice it does not pose a problem. For a single unit of JD returns

$$
\chi(s)=\frac{s^{2}}{2}+\eta\left(e^{s^{2} / 2}-1\right)-s \mu .
$$

For a single unit of TL returns with positive drift $\mu$

$\chi(s)=-s \mu-\frac{c^{\alpha}}{\cos (\pi \alpha / 2)}\left(\left(\beta^{2}-s^{2}\right)^{\alpha / 2} \frac{\left(\left(1-\frac{s}{\beta}\right)^{\frac{\alpha}{2}}\left(1+\frac{s}{\beta}\right)^{\frac{-\alpha}{2}}+\left(1-\frac{s}{\beta}\right)^{\frac{-\alpha}{2}}\left(1+\frac{s}{\beta}\right)^{\frac{\alpha}{2}}\right)}{2}-\beta^{\alpha}\right)$.

As an illustration, for a single unit of JD process, Figure 7 shows $\delta$ and $\chi^{\prime}(\delta)$ as a function of the mean jump inter-arrival time $1 / \eta$. Note that as $\eta \rightarrow 0, \delta \rightarrow 2 \mu$, as can be seen in the graph. Note that the rescaled time to an unacceptable loss $\chi^{\prime}(\delta)$ behaves quite differently to the exponent $\delta$.

\section{References}

[1] Brigitte C. Madrian and Dennis F. Shea. The power of suggestion: Inertia in 401(k) participation and savings behavior. Quarterly Journal of Economics, 116(4):1149-1197, 2001. 
[2] James J. Choi, David Laibson, Brigitte C. Madrian, and Andrew Metrick. Defined contribution pensions: Plan rules, participant decisions, and the path of least resistance. In James M. Poterba, editor, Tax Policy and the Economy, volume 16, pages 67-113. MIT Press, 2002.

[3] James J. Choi, David Laibson, Brigitte C. Madrian, and Andrew Metrick. Saving for retirement on the path of least resistance. In Ed McCaffrey and Joel Slemrod, editors, Behavioral Public Finance: Toward a New Agenda. New York: Russell Sage Foundation, 2006.

[4] F. P. Kelly. Notes on effective bandwidths. In F. P. Kelly, S. Zachary, and I. B. Ziedins, editors, Stochastic Networks: Theory and Applications. Oxford University Press, 1996.

[5] Patrick Billingsley. Probability and measure. Wiley Series in Probability and Mathematical Statistics. John Wiley \& Sons Inc., New York, third edition, 1995.

[6] A. J. Ganesh and Neil O'Connell. A large deviation principle with queueing applications. Stoch. Stoch. Rep., 73(1-2):25-35, 2002.

[7] Ken Duffy, John T. Lewis, and Wayne G. Sullivan. Logarithmic asymptotics for the supremum of a stochastic process. Ann. Appl. Probab., 13(2):430-445, 2003.

[8] Amir Dembo and Tim Zajic. Large deviations: from empirical mean and measure to partial sums process. Stochastic Process. Appl., 57(2):191-224, 1995.

[9] A. N. Kolmogorov. Wienersche Spiralen und einige andere interessante Kurven im Hilbertschen Raum. C. R. (Doklady) Acad. Sci. URSS (N.S.), 26:115-118, 1940.

[10] Benoit B. Mandelbrot and John W. Van Ness. Fractional Brownian motions, fractional noises and applications. SIAM Rev., 10:422-437, 1968.

[11] Gennady Samorodnitsky and Murad S. Taqqu. Stable non-Gaussian random processes. Stochastic Modeling. Chapman \& Hall, New York, 1994. Stochastic models with infinite variance.

[12] R. C. Merton. Option pricing when underlying stock returns are discontinuous. Journal of Financial Economics, 3:125-144, 1976.

[13] D.P. Madan, P. Carr, and E. Chang. The variance gamma process and option pricing. European Finance Review, 2(1):79-105, 1998.

[14] William Feller. An introduction to probability theory and its applications. Vol. II. Second edition. John Wiley \& Sons Inc., New York, 1971.

[15] Rosario N. Mantegna and H. Eugene Stanley. Stochastic process with ultraslow convergence to a Gaussian: the truncated Lévy flight. Phys. Rev. Lett., 73(22):2946-2949, 1994. 
[16] Andrew Matacz. Financial modeling and option theory with the truncated Lévy process. International Journal of Theoretical and Applied Finance, 3(1):143-160, 2000.

[17] Ismo Koponen. Analytic approach to the problem of convergence of truncated Lévy flights towards the gaussian stochastic process. Phys. Rev. E, 52(1):1197-1199, Jul 1995. 